Supporting information

\title{
Carrier-Envelope-Phase Modulated Currents in Scanning Tunneling Microscopy
}

\author{
Ziyang Hu, ${ }^{\dagger}$ YanHo Kwok, ${ }^{\dagger, *}$ GuanHua Chen, ${ }^{*}, \dagger, \| l$ and Shaul Mukamel ${ }^{*}, \S$ \\ 'Department of Chemistry, The University of Hong Kong, Pokfulam, Hong Kong SAR \\ QuantumFabless Limited, Sha Tin, Hong Kong SAR \\ "Hong Kong Quantum AI Lab Limited, Pak Shek Kok, Hong Kong SAR
}

${ }^{\S}$ Department of Chemistry and Physics \& Astronomy, University of California, Irvine, California 92617, United States

*Email: ghc@everest.hku.hk (G.C.)

*Email: smukamel@uci.edu (S.M.) 


\section{First Order Integrated Current}

For simplicity, we use the following abbreviated integration notation,

$$
\int \mathrm{d} t_{1} \mathrm{~d} t_{2} \cdots \mathrm{d} t_{n} \mathbf{A}_{0}\left(t, t_{1}\right) \mathbf{A}_{1}\left(t_{1}, t_{2}\right) \cdots \mathbf{A}_{n}\left(t_{n}, t\right)=\int \mathrm{d} \tau \mathbf{A}_{0} \mathbf{A}_{1} \cdots \mathbf{A}_{n} .
$$

$\mathbf{A}_{i}$ are general matrices, and they can also be single-variable, $\mathbf{A}_{i}\left(t_{i}\right)$. The fold of integration can be deduced from the number of $\mathbf{A}_{i}$.

We split the response to electric field into two parts, i.e., (1) the sole response to induced bias; (2) the remaining part. The first part is

$$
I_{\alpha}^{(1,1)}(t)=e \int \mathrm{d} \tau \operatorname{tr}\left[\mathbf{G}^{r} \boldsymbol{\Sigma}_{1}^{<} \mathbf{G}^{a} \boldsymbol{\Sigma}_{\alpha}^{a}+\mathbf{G}^{r} \boldsymbol{\Sigma}_{\alpha 1}^{<}+\mathbf{h . c} .\right]
$$

Perform Fourier transform from $t$ to $\omega$,

$$
\begin{aligned}
\mathcal{F}_{t}\left[\int \mathrm{d} \tau \mathbf{G}^{r} \boldsymbol{\Sigma}_{1}^{<} \mathbf{G}^{a} \boldsymbol{\Sigma}_{\alpha}^{a}\right] & =\sum_{\beta} \frac{e V_{\beta}(\omega)}{2 \pi \hbar^{2} \omega} \int \mathrm{d} \varepsilon \mathbf{G}_{+}^{r} \boldsymbol{\Sigma}_{\beta}^{<} \mathbf{G}^{a} \boldsymbol{\Sigma}_{\alpha}^{a}, \\
\mathcal{F}_{t}\left[\int \mathrm{d} \tau \mathbf{G}^{r} \boldsymbol{\Sigma}_{\alpha 1}^{<}\right] & =\frac{e V_{\alpha}(\omega)}{2 \pi \hbar^{2} \omega} \int \mathrm{d} \varepsilon \mathbf{G}_{+}^{r} \boldsymbol{\Sigma}_{\alpha}^{<} .
\end{aligned}
$$

Notations $\mathbf{A}=\mathbf{A}(\varepsilon), \mathbf{A}_{+}=\mathbf{A}(\varepsilon+\hbar \omega), \underset{\mathbf{A}}{=} \mathbf{A}-\mathbf{A}_{+}$are used in eq S3. Choose $V_{\alpha}(t)=E(t) L / 2$ and $V_{\beta}(t)=-E(t) L / 2$, we have

$$
\begin{aligned}
I_{\alpha}^{(1,1)}(\omega) & =\frac{e E(\omega)}{2 \pi \hbar} \sum_{\beta} \int \mathrm{d} \varepsilon \operatorname{tr}\left[\mathcal{G}_{\alpha \beta}^{(1,1)}(\varepsilon, \omega)\right], \\
\mathcal{G}_{\alpha \beta}^{(1,1)}(\varepsilon, \omega) & =\frac{e L}{2 \hbar \omega}\left[\left(2 \delta_{\alpha \beta}-1\right)\left(\mathbf{G}_{+}^{r} \boldsymbol{\Sigma}_{\beta}^{<} \mathbf{G}^{a} \boldsymbol{\Sigma}_{\alpha}^{a}-\boldsymbol{\Sigma}_{\alpha}^{r} \mathbf{G}_{+}^{r} \boldsymbol{\Sigma}_{\beta}^{<} \mathbf{G}^{a}\right)+\delta_{\alpha \beta}\left(\mathbf{G}_{+}^{r} \boldsymbol{\Sigma}_{\beta}^{<}-\boldsymbol{\Sigma}_{\beta}^{<} \mathbf{G}^{a}\right)\right] .
\end{aligned}
$$

The second part is 


$$
I_{\alpha}^{(1,2)}(t)=e \int \mathrm{d} \tau \operatorname{tr}\left[\mathbf{G}^{r} \Delta \mathbf{G}^{<} \boldsymbol{\Sigma}_{\alpha}^{a}+\mathbf{G}^{<} \Delta \mathbf{G}^{a} \boldsymbol{\Sigma}_{\alpha}^{a}+\mathbf{G}^{r} \Delta \mathbf{G}^{r} \boldsymbol{\Sigma}_{\alpha}^{<}+\text {h.c. }\right] .
$$

Perform Fourier transform from $t$ to $\omega$,

$$
I_{\alpha}^{(1,2)}(\omega)=\frac{e}{2 \pi \hbar} \int \mathrm{d} \varepsilon \operatorname{tr}\left[\mathbf{G}_{+}^{r} \Delta(\omega) \mathbf{G}^{<} \boldsymbol{\Sigma}_{\alpha}^{a}+\mathbf{G}_{+}^{<} \Delta(\omega) \mathbf{G}^{a} \boldsymbol{\Sigma}_{\alpha}^{a}+\mathbf{G}_{+}^{r} \Delta(\omega) \mathbf{G}^{r} \boldsymbol{\Sigma}_{\alpha}^{<}+\text {h.c. }\right] .
$$

Set $\omega=0$ and denote $\boldsymbol{\Lambda}_{\alpha}=\operatorname{Im}\left[\boldsymbol{\Sigma}_{\alpha}^{a}\right]$, we have

$$
\begin{aligned}
J_{\alpha}^{(1,2)} & =\frac{i e}{\pi \hbar} \int \mathrm{d} \varepsilon \operatorname{tr}\left[\Delta(\omega=0)\left(\mathbf{G}^{<} \boldsymbol{\Lambda}_{\alpha} \mathbf{G}^{r}+\mathbf{G}^{a} \boldsymbol{\Lambda}_{\alpha} \mathbf{G}^{<}+F \mathbf{G}^{r} \boldsymbol{\Lambda}_{\alpha} \mathbf{G}^{r}-F \mathbf{G}^{a} \boldsymbol{\Lambda}_{\alpha} \mathbf{G}^{a}\right)\right] \\
& =0 .
\end{aligned}
$$

$F(\varepsilon)$ is the Fermi-Dirac distribution, and $\mathbf{G}^{<}=-F\left[\mathbf{G}^{r}-\mathbf{G}^{a}\right]$ is used in the last line of eq S7.

The first order integrated current is

$$
\begin{aligned}
J_{\alpha}^{(1)} & =\frac{e E(\omega=0)}{2 \pi \hbar} \sum_{\beta} \int \mathrm{d} \varepsilon \operatorname{tr}\left[\mathcal{G}_{\alpha \beta}^{(1)}(\varepsilon)\right], \\
\mathcal{G}_{\alpha \beta}^{(1)}(\varepsilon) & =\lim _{\omega \rightarrow 0} \mathcal{G}_{\alpha \beta}^{(1,1)}(\varepsilon, \omega) .
\end{aligned}
$$

\section{Second Order Integrated Current}

Following the split scheme used in the first order integrated current, the first part of second order current is

$$
I_{\alpha}^{(2,1)}(t)=e \int \mathrm{d} \tau \operatorname{tr}\left[\mathbf{G}^{r} \boldsymbol{\Sigma}_{2}^{<} \mathbf{G}^{a} \boldsymbol{\Sigma}_{\alpha}^{a}+\mathbf{G}^{r} \boldsymbol{\Sigma}_{\alpha 2}^{<}+\text {h.c. }\right] .
$$

Perform Fourier transform from $t$ to $\omega$ and set $\omega=0$, 


$$
\begin{gathered}
J_{\alpha}^{(2,1)}=\frac{e}{4 \pi^{2} \hbar^{2}} \sum_{\beta} \int \mathrm{d} \varepsilon_{1} E\left(-\varepsilon_{1} / \hbar\right) E\left(\varepsilon_{1} / \hbar\right) \int \mathrm{d} \varepsilon \operatorname{tr}\left[\mathcal{G}_{\alpha \beta}^{(2,1)}\left(\varepsilon, \varepsilon_{1}\right)\right] \\
\mathcal{G}_{\alpha \beta}^{(2,1)}\left(\varepsilon, \varepsilon_{1}\right)=-\frac{e^{2} L^{2}}{4 \varepsilon_{1}^{2}}\left[\mathbf{G}^{r} \underline{\boldsymbol{\Sigma}}_{\beta}^{<} \mathbf{G}^{a} \boldsymbol{\Sigma}_{\alpha}^{a}-\boldsymbol{\Sigma}_{\alpha}^{r} \mathbf{G}^{r} \underline{\boldsymbol{\Sigma}}_{\beta}^{<} \mathbf{G}^{a}+\delta_{\alpha \beta}\left(\mathbf{G}^{r} \underline{\boldsymbol{\Sigma}}_{\beta}^{<}-\underline{\boldsymbol{\Sigma}}_{\beta}^{<} \mathbf{G}^{a}\right)\right] .
\end{gathered}
$$

Notation $\underline{\mathbf{A}}=\mathbf{A}-\mathbf{A}\left(\varepsilon+\varepsilon_{1}\right)$ is used. Plug the relation $\boldsymbol{\Sigma}_{\alpha}^{<}(\varepsilon)=2 i \boldsymbol{\Lambda}_{\alpha} F(\varepsilon)$ into eq S10, it can be shown that

$$
J_{\alpha}^{(2,1)}=0
$$

The second part is

$$
\begin{aligned}
I_{\alpha}^{(2,2)}(t) & =e \int \mathrm{d} \tau \operatorname{tr}\left[\mathbf{G}^{r} \Delta \mathbf{G}^{r} \Delta \mathbf{G}^{<} \boldsymbol{\Sigma}_{\alpha}^{a}+\mathbf{G}^{<} \boldsymbol{\Delta} \mathbf{G}^{a} \Delta \mathbf{G}^{a} \boldsymbol{\Sigma}_{\alpha}^{a}\right. \\
& +\mathbf{G}^{r} \boldsymbol{\Delta} \mathbf{G}^{<} \boldsymbol{\Delta} \mathbf{G}^{a} \boldsymbol{\Sigma}_{\alpha}^{a}+\mathbf{G}^{r} \boldsymbol{\Delta} \mathbf{G}^{r} \Delta \mathbf{G}^{r} \boldsymbol{\Sigma}_{\alpha}^{<}+\mathbf{G}^{r} \Delta \mathbf{G}^{r} \boldsymbol{\Sigma}_{\alpha 1}^{<} \\
& \left.+\mathbf{G}^{r} \boldsymbol{\Delta} \mathbf{G}^{r} \boldsymbol{\Sigma}_{1}^{<} \mathbf{G}^{a} \boldsymbol{\Sigma}_{\alpha}^{a}+\mathbf{G}^{r} \boldsymbol{\Sigma}_{1}^{<} \mathbf{G}^{a} \Delta \mathbf{G}^{a} \boldsymbol{\Sigma}_{\alpha}^{a}+\text { h.c. }\right] .
\end{aligned}
$$

Perform Fourier transform from $t$ to $\omega$ and set $\omega=0$,

$$
\begin{aligned}
J_{\alpha}^{(2,2)}= & \frac{e}{4 \pi^{2} \hbar^{2}} \sum_{\beta} \int \mathrm{d} \varepsilon_{1} E\left(-\varepsilon_{1} / \hbar\right) E\left(\varepsilon_{1} / \hbar\right) \int \mathrm{d} \varepsilon \operatorname{tr}\left[\mathcal{G}_{\alpha \beta}^{(2,2)}\left(\varepsilon, \varepsilon_{1}\right)\right], \\
\mathcal{G}_{\alpha \beta}^{(2,2)}\left(\varepsilon, \varepsilon_{1}\right)= & \delta_{\alpha \beta}\left(\mathbf{G}^{r} \boldsymbol{\mu} \mathbf{G}_{+1}^{r} \boldsymbol{\mu} \mathbf{G}^{<} \boldsymbol{\Sigma}_{\alpha}^{a}+\mathbf{G}^{<} \boldsymbol{\mu} \mathbf{G}_{+1}^{a} \boldsymbol{\mu} \mathbf{G}^{a} \boldsymbol{\Sigma}_{\alpha}^{a}\right. \\
& \left.+\mathbf{G}^{r} \boldsymbol{\mu} \mathbf{G}_{+1}^{<} \boldsymbol{\mu} \mathbf{G}^{a} \boldsymbol{\Sigma}_{\alpha}^{a}+\mathbf{G}^{r} \boldsymbol{\mu} \mathbf{G}_{+1}^{r} \boldsymbol{\mu} \mathbf{G}^{r} \boldsymbol{\Sigma}_{\alpha}^{<}-\frac{e L}{2 \varepsilon_{1}} \mathbf{G}^{r} \boldsymbol{\mu} \mathbf{G}_{+1}^{r} \underline{\Sigma}_{\alpha}^{<}\right) \\
& -\frac{e \boldsymbol{L}}{2 \varepsilon_{1}}\left(2 \delta_{\alpha \beta}-1\right)\left(\mathbf{G}^{r} \boldsymbol{\mu} \mathbf{G}_{+1}^{r} \underline{\Sigma}_{\beta}^{<} \mathbf{G}^{a} \boldsymbol{\Sigma}_{\alpha}^{a}+\mathbf{G}^{r} \underline{\boldsymbol{\Sigma}}_{\beta}^{<} \mathbf{G}_{+1}^{a} \boldsymbol{\mu} \mathbf{G}^{a} \boldsymbol{\Sigma}_{\alpha}^{a}\right) \\
& + \text { h.c. }
\end{aligned}
$$

Notations $\mathbf{A}_{+1}=\mathbf{A}\left(\varepsilon+\varepsilon_{1}\right), \underline{\mathbf{A}}=\mathbf{A}-\mathbf{A}_{+1}$ are used.

The second order integrated current is 


$$
\begin{aligned}
J_{\alpha}^{(2)} & =\frac{e}{4 \pi^{2} \hbar^{2}} \sum_{\beta} \int \mathrm{d} \varepsilon_{1} E\left(-\varepsilon_{1} / \hbar\right) E\left(\varepsilon_{1} / \hbar\right) \int \mathrm{d} \varepsilon \operatorname{tr}\left[\mathcal{G}_{\alpha \beta}^{(2)}\left(\varepsilon, \varepsilon_{1}\right)\right], \\
\mathcal{G}_{\alpha \beta}^{(2)}\left(\varepsilon, \varepsilon_{1}\right) & =\mathcal{G}_{\alpha \beta}^{(2,2)}\left(\varepsilon, \varepsilon_{1}\right) .
\end{aligned}
$$

\section{Third Order Integrated Current}

Following the split scheme used in the first order integrated current, the first part of third order current is

$$
I_{\alpha}^{(3,1)}(t)=e \int \mathrm{d} \tau \operatorname{tr}\left[\mathbf{G}^{r} \boldsymbol{\Sigma}_{3}^{<} \mathbf{G}^{a} \boldsymbol{\Sigma}_{\alpha}^{a}+\mathbf{G}^{r} \boldsymbol{\Sigma}_{\alpha 3}^{<}+\text {h.c. }\right]
$$

Perform Fourier transform from $t$ to $\omega$ and set $\omega=0$,

$$
\begin{aligned}
& J_{\alpha}^{(3,1)}=\frac{e}{8 \pi^{3} \hbar^{3}} \sum_{\beta} \iint \mathrm{d} \varepsilon_{2} \mathrm{~d} \varepsilon_{1} E\left(\frac{\varepsilon_{1}}{\hbar}\right) E\left(\frac{\varepsilon_{2}-\varepsilon_{1}}{\hbar}\right) E\left(-\frac{\varepsilon_{2}}{\hbar}\right) \int \mathrm{d} \varepsilon \operatorname{tr}\left[\mathcal{G}_{\alpha \beta}^{(3,1)}\left(\varepsilon, \varepsilon_{1}, \varepsilon_{2}\right)\right], \\
& \mathcal{G}_{\alpha \beta}^{(3,1)}\left(\varepsilon, \varepsilon_{1}, \varepsilon_{2}\right)=\frac{e^{3} L^{3}}{16 \varepsilon_{1}\left(\varepsilon_{1}-\varepsilon_{2}\right) \varepsilon_{2}}\left[\left(2 \delta_{\alpha \beta}-1\right)\left(\mathbf{G}^{r} \underset{\sim \beta}{\boldsymbol{\Sigma}_{\alpha}^{<}} \mathbf{G}^{a} \boldsymbol{\Sigma}_{\alpha}^{a}-\boldsymbol{\Sigma}_{\alpha}^{r} \mathbf{G}^{r} \underset{\sim \beta}{\boldsymbol{\Sigma}_{\beta}^{<}} \mathbf{G}^{a}\right)+\delta_{\alpha \beta}\left(\mathbf{G}^{r} \underset{\sim \beta}{\boldsymbol{\Sigma}_{\beta}^{<}}-\underset{\sim \beta}{\boldsymbol{\Sigma}_{\beta}^{<}} \mathbf{G}^{a}\right)\right] .
\end{aligned}
$$

Notation $\underset{\sim}{\mathbf{A}}=\mathbf{A}\left(\varepsilon+\varepsilon_{1}\right)-\mathbf{A}\left(\varepsilon+\varepsilon_{2}\right)$ is used. The second part is

$$
\begin{aligned}
& I_{\alpha}^{(3,2)}(t)=e \int \mathrm{d} \tau \operatorname{tr}\left[\mathbf{G}^{r} \Delta \mathbf{G}^{r} \Delta \mathbf{G}^{r} \Delta \mathbf{G}^{<} \boldsymbol{\Sigma}_{\alpha}^{a}+\mathbf{G}^{<} \boldsymbol{\Delta} \mathbf{G}^{a} \Delta \mathbf{G}^{a} \boldsymbol{\Delta} \mathbf{G}^{a} \boldsymbol{\Sigma}_{\alpha}^{a}\right. \\
& +\mathbf{G}^{r} \Delta \mathbf{G}^{r} \Delta \mathbf{G}^{<} \Delta \mathbf{G}^{a} \boldsymbol{\Sigma}_{\alpha}^{a}+\mathbf{G}^{r} \Delta \mathbf{G}^{<} \Delta \mathbf{G}^{a} \Delta \mathbf{G}^{a} \boldsymbol{\Sigma}_{\alpha}^{a} \\
& +\mathbf{G}^{r} \Delta \mathbf{G}^{r} \Delta \mathbf{G}^{r} \Delta \mathbf{G}^{r} \boldsymbol{\Sigma}_{\alpha}^{<}+\mathbf{G}^{r} \Delta \mathbf{G}^{r} \Delta \mathbf{G}^{r} \boldsymbol{\Sigma}_{\alpha 1}^{<} \\
& +\mathbf{G}^{r} \Delta \mathbf{G}^{r} \Delta \mathbf{G}^{r} \boldsymbol{\Sigma}_{1}^{<} \mathbf{G}^{a} \boldsymbol{\Sigma}_{\alpha}^{a}+\mathbf{G}^{r} \Sigma_{1}^{<} \mathbf{G}^{a} \Delta \mathbf{G}^{a} \Delta \mathbf{G}^{a} \boldsymbol{\Sigma}_{\alpha}^{a} \\
& +\mathbf{G}^{r} \Delta \mathbf{G}^{r} \boldsymbol{\Sigma}_{1}^{<} \mathbf{G}^{a} \boldsymbol{\Delta} \mathbf{G}^{a} \boldsymbol{\Sigma}_{\alpha}^{a}+\mathbf{G}^{r} \Delta \mathbf{G}^{r} \boldsymbol{\Sigma}_{\alpha_{2}}^{<} \\
& \left.+\mathbf{G}^{r} \Delta \mathbf{G}^{r} \boldsymbol{\Sigma}_{2}^{<} \mathbf{G}^{a} \boldsymbol{\Sigma}_{\alpha}^{a}+\mathbf{G}^{r} \boldsymbol{\Sigma}_{2}^{<} \mathbf{G}^{a} \Delta \mathbf{G}^{a} \boldsymbol{\Sigma}_{\alpha}^{a}+\text { h.c. }\right] .
\end{aligned}
$$

Perform Fourier transform from $t$ to $\omega$ and set $\omega=0$, 


$$
\begin{aligned}
& J_{\alpha}^{(3,2)}=\frac{e}{8 \pi^{3} \hbar^{3}} \sum_{\beta} \iint \mathrm{d} \varepsilon_{2} \mathrm{~d} \varepsilon_{1} E\left(\frac{\varepsilon_{1}}{\hbar}\right) E\left(\frac{\varepsilon_{2}-\varepsilon_{1}}{\hbar}\right) E\left(-\frac{\varepsilon_{2}}{\hbar}\right) \int \mathrm{d} \varepsilon \operatorname{tr}\left[\mathcal{G}_{\alpha \beta}^{(3,2)}\left(\varepsilon, \varepsilon_{1}, \varepsilon_{2}\right)\right], \\
& \mathcal{G}_{\alpha \beta}^{(3,2)}\left(\varepsilon, \varepsilon_{1}, \varepsilon_{2}\right)=-\delta_{\alpha \beta}\left(\mathbf{G}^{r} \boldsymbol{\mu} \mathbf{G}_{+2}^{r} \boldsymbol{\mu} \mathbf{G}_{+1}^{r} \boldsymbol{\mu} \mathbf{G}^{<} \boldsymbol{\Sigma}_{\alpha}^{a}-\boldsymbol{\Sigma}_{\alpha}^{r} \mathbf{G}^{<} \boldsymbol{\mu} \mathbf{G}_{+2}^{a} \boldsymbol{\mu} \mathbf{G}_{+1}^{a} \boldsymbol{\mu} \mathbf{G}^{a}\right) \\
& -\delta_{\alpha \beta}\left(\mathbf{G}^{<} \boldsymbol{\mu} \mathbf{G}_{+2}^{a} \boldsymbol{\mu} \mathbf{G}_{+1}^{a} \boldsymbol{\mu} \mathbf{G}^{a} \boldsymbol{\Sigma}_{\alpha}^{a}-\boldsymbol{\Sigma}_{\alpha}^{r} \mathbf{G}^{r} \boldsymbol{\mu} \mathbf{G}_{+2}^{r} \boldsymbol{\mu} \mathbf{G}_{+1}^{r} \boldsymbol{\mu} \mathbf{G}^{<}\right) \\
& -\delta_{\alpha \beta}\left(\mathbf{G}^{r} \boldsymbol{\mu} \mathbf{G}_{+2}^{r} \boldsymbol{\mu} \mathbf{G}_{+1}^{<} \boldsymbol{\mu} \mathbf{G}^{a} \boldsymbol{\Sigma}_{\alpha}^{a}-\boldsymbol{\Sigma}_{\alpha}^{r} \mathbf{G}^{r} \boldsymbol{\mu} \mathbf{G}_{+2}^{<} \boldsymbol{\mu} \mathbf{G}_{+1}^{a} \boldsymbol{\mu} \mathbf{G}^{a}\right) \\
& -\delta_{\alpha \beta}\left(\mathbf{G}^{r} \boldsymbol{\mu} \mathbf{G}_{+2}^{<} \boldsymbol{\mu} \mathbf{G}_{+1}^{a} \boldsymbol{\mu} \mathbf{G}^{a} \boldsymbol{\Sigma}_{\alpha}^{a}-\boldsymbol{\Sigma}_{\alpha}^{r} \mathbf{G}^{r} \boldsymbol{\mu} \mathbf{G}_{+2}^{r} \boldsymbol{\mu} \mathbf{G}_{+1}^{<} \boldsymbol{\mu} \mathbf{G}^{a}\right) \\
& -\delta_{\alpha \beta}\left(\mathbf{G}^{r} \boldsymbol{\mu} \mathbf{G}_{+2}^{r} \boldsymbol{\mu} \mathbf{G}_{+1}^{r} \boldsymbol{\mu} \mathbf{G}^{r} \boldsymbol{\Sigma}_{\alpha}^{<}-\boldsymbol{\Sigma}_{\alpha}^{<} \mathbf{G}^{a} \boldsymbol{\mu} \mathbf{G}_{+2}^{a} \boldsymbol{\mu} \mathbf{G}_{+1}^{a} \boldsymbol{\mu} \mathbf{G}^{a}\right) \\
& +\frac{e L}{2} \delta_{\alpha \beta}\left[\frac{1}{\varepsilon_{1}} \mathbf{G}^{r} \boldsymbol{\mu} \mathbf{G}_{+2}^{r} \boldsymbol{\mu} \mathbf{G}_{+1}^{r} \underline{\Sigma}_{\alpha}^{<}-\frac{1}{\varepsilon_{2}} \stackrel{\boldsymbol{\Sigma}}{=}_{\alpha}^{<} \mathbf{G}_{+2}^{a} \boldsymbol{\mu} \mathbf{G}_{+1}^{a} \boldsymbol{\mu} \mathbf{G}^{a}\right] \\
& +\frac{e L}{2}\left(2 \delta_{\alpha \beta}-1\right)\left[\frac{1}{\varepsilon_{1}} \mathbf{G}^{r} \boldsymbol{\mu} \mathbf{G}_{+2}^{r} \boldsymbol{\mu} \mathbf{G}_{+1}^{r} \underline{\Sigma}_{\beta}^{<} \mathbf{G}^{a} \boldsymbol{\Sigma}_{\alpha}^{a}-\frac{1}{\varepsilon_{2}} \boldsymbol{\Sigma}_{\alpha}^{r} \mathbf{G}^{r} \underline{=}_{\beta}^{<} \mathbf{G}_{+2}^{a} \boldsymbol{\mu} \mathbf{G}_{+1}^{a} \boldsymbol{\mu} \mathbf{G}^{a}\right] \\
& +\frac{e L}{2}\left(2 \delta_{\alpha \beta}-1\right)\left[\frac{1}{\varepsilon_{2}} \mathbf{G}^{r} \underline{\underline{\Sigma}}_{\beta}^{<} \mathbf{G}_{+2}^{a} \boldsymbol{\mu} \mathbf{G}_{+1}^{a} \boldsymbol{\mu} \mathbf{G}^{a} \boldsymbol{\Sigma}_{\alpha}^{a}-\frac{1}{\varepsilon_{1}} \boldsymbol{\Sigma}_{\alpha}^{r} \mathbf{G}^{r} \boldsymbol{\mu} \mathbf{G}_{+2}^{r} \boldsymbol{\mu} \mathbf{G}_{+1}^{r} \underline{\boldsymbol{\Sigma}}_{\beta}^{<} \mathbf{G}^{a}\right] \\
& +\frac{e L\left(2 \delta_{\alpha \beta}-1\right)}{2\left(\varepsilon_{2}-\varepsilon_{1}\right)}\left[\mathbf{G}^{r} \boldsymbol{\mu} \mathbf{G}_{+2}^{r} \underset{\sim \beta}{\boldsymbol{\Sigma}_{\beta}^{<}} \mathbf{G}_{+1}^{a} \boldsymbol{\mu} \mathbf{G}^{a} \boldsymbol{\Sigma}_{\alpha}^{a}-\boldsymbol{\Sigma}_{\alpha}^{r} \mathbf{G}^{r} \boldsymbol{\mu} \mathbf{G}_{+2}^{r} \underset{\sim \beta}{\boldsymbol{\Sigma}_{\alpha}^{<}} \mathbf{G}_{+1}^{a} \boldsymbol{\mu} \mathbf{G}^{a}\right] \\
& +\frac{e^{2} L^{2} \delta_{\alpha \beta}}{8\left(\varepsilon_{2}-\varepsilon_{1}\right)}\left[\frac{1}{\varepsilon_{1}} \mathbf{G}^{r} \boldsymbol{\mu} \mathbf{G}_{+2}^{r}\left(\underset{\sim \alpha}{\boldsymbol{\Sigma}}-\underline{\boldsymbol{\Sigma}}_{\alpha}^{<}\right)-\frac{1}{\varepsilon_{2}}\left(\underset{\sim \alpha}{\boldsymbol{\Sigma}}+\underset{\underline{\boldsymbol{\Sigma}_{\alpha}}}{<}\right) \mathbf{G}_{+1}^{a} \boldsymbol{\mu} \mathbf{G}^{a}\right] \\
& +\frac{e^{2} L^{2}}{8\left(\varepsilon_{2}-\varepsilon_{1}\right)}\left[\frac{1}{\varepsilon_{1}} \mathbf{G}^{r} \boldsymbol{\mu} \mathbf{G}_{+2}^{r}\left(\underset{\sim \beta}{\boldsymbol{\Sigma}_{\beta}^{<}}-\underline{\boldsymbol{\Sigma}}_{\beta}^{<}\right) \mathbf{G}^{a} \boldsymbol{\Sigma}_{\alpha}^{a}-\frac{1}{\varepsilon_{2}} \boldsymbol{\Sigma}_{\alpha}^{r} \mathbf{G}^{r}\left(\underset{\sim \beta}{\boldsymbol{\Sigma}}+\underset{\boldsymbol{\Sigma}_{\beta}^{<}}{\boldsymbol{\Sigma}^{<}}\right) \mathbf{G}_{+1}^{a} \boldsymbol{\mu} \mathbf{G}^{a}\right] \\
& +\frac{e^{2} L^{2}}{8\left(\varepsilon_{2}-\varepsilon_{1}\right)}\left[\frac{1}{\varepsilon_{2}} \mathbf{G}^{r}\left(\underset{\sim \beta}{\boldsymbol{\Sigma}_{\beta}^{<}}+\underset{=}{\boldsymbol{\Sigma}_{\beta}^{<}}\right) \mathbf{G}_{+1}^{a} \boldsymbol{\mu} \mathbf{G}^{a} \boldsymbol{\Sigma}_{\alpha}^{a}-\frac{1}{\varepsilon_{1}} \boldsymbol{\Sigma}_{\alpha}^{r} \mathbf{G}^{r} \boldsymbol{\mu} \mathbf{G}_{+2}^{r}\left(\underset{\sim \beta}{\boldsymbol{\Sigma}_{\beta}^{<}}-\underline{\boldsymbol{\Sigma}}_{\beta}^{<}\right) \mathbf{G}^{a}\right] \text {. }
\end{aligned}
$$

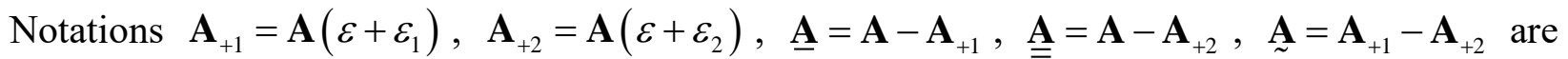
used.

The third order integrated current is 


$$
\begin{aligned}
J_{\alpha}^{(3)} & =\frac{e}{8 \pi^{3} \hbar^{3}} \sum_{\beta} \iint \mathrm{d} \varepsilon_{2} \mathrm{~d} \varepsilon_{1} E\left(\frac{\varepsilon_{1}}{\hbar}\right) E\left(\frac{\varepsilon_{2}-\varepsilon_{1}}{\hbar}\right) E\left(-\frac{\varepsilon_{2}}{\hbar}\right) \int \mathrm{d} \varepsilon \operatorname{tr}\left[\mathcal{G}_{\alpha \beta}^{(3)}\left(\varepsilon, \varepsilon_{1}, \varepsilon_{2}\right)\right], \\
\mathcal{G}_{\alpha \beta}^{(3)}\left(\varepsilon, \varepsilon_{1}, \varepsilon_{2}\right) & =\mathcal{G}_{\alpha \beta}^{(3,1)}\left(\varepsilon, \varepsilon_{1}, \varepsilon_{2}\right)+\mathcal{G}_{\alpha \beta}^{(3,2)}\left(\varepsilon, \varepsilon_{1}, \varepsilon_{2}\right) .
\end{aligned}
$$

